\title{
¿PUEDE EL SER HUMANO ALCANZAR LA FELICIDAD?
}

\author{
Sonia María Rodríguez Huerta \\ Universidad de Oviedo \\ soniamariarodriguez9@gmail.com
}

Recepción Artículo: 15 enero 2020

Admisión Evaluación: 4 marzo 2020

Informe Evaluador 1: 17 marzo 2020

Informe Evaluador 2: 15 marzo 2020

Aprobación Publicación: 20 abril 2020

\section{RESUMEN}

El ser humano mueve las piezas de su propia vida para llegar a conseguir su felicidad. En el trabajo se cuestiona radicalmente la propia posibilidad de consecución de la felicidad, partiendo de una especie de agnosia sobre su concepción como estadio final. Las ideas homologadas de la felicidad siguen vivas en nuestros días, cada vez más y en más disciplinas, sobre todo en las sociedades occidentales (Bueno, 2011). Se llama la atención sobre el aspecto bifronte de la felicidad, físico, por un lado, y espiritual por otro. Se concluye que es muy difícil que podamos alcanzar el grado máximo y permanente de felicidad o la felicidad completa. Puede que asumamos el peso y la responsabilidad de conseguir la máxima felicidad pero, como Sísifo, cargando una y otra vez la gigantesca piedra hacia la cumbre de la montaña, nunca podamos mantenernos allí, en ese territorio ideal o emocional del que poco conocemos y que se configura como una asíntota. Con respecto a la posibilidad de ser feliz en mayor o menor medida, es sugerente la propuesta de la pirámide gradual de Abraham Maslow. La conclusión es optimista, al entender que la felicidad relativa engloba un rango muy variable y no es un punto final. Se propone la modificación del significado del término "felicidad" en el Diccionario de la Real Academia Española de la Lengua, para identificarlo con "una variedad de estados de grata satisfacción espiritual y física".

Palabras clave: felicidad; pirámide gradual; Maslow

\section{ABSTRACT}

Can human beings achieve happiness? The human being moves the pieces of his own life to achieve his happiness. In the work, the very possibility of achieving happiness is radically questioned, starting from a kind of agnosia about its conception as a final stage. The approved ideas of happiness are still alive in our day, increasingly and in more disciplines, especially in Western societies (Bueno, 2011). It draws attention to the two-sided aspect of happiness, physical, on the one hand, and spiritual on the other. It is concluded that it is very difficult for us to achieve the maximum and permanent degree of happiness or complete happiness. We may assume the 
weight and responsibility of achieving maximum happiness but, like Sisyphus, carrying the gigantic stone to the top of the mountain over and over again, we can never stay there, in that ideal or emotional territory that we know little about and that is shaped like an asymptote. With regard to the possibility of being happier to a greater or lesser extent, the proposal of Abraham Maslow's gradual pyramid is suggestive. The conclusion is optimistic, understanding that relative happiness encompasses a very variable range and is not an end point. It is proposed to modify the meaning of the term "happiness" in the Dictionary of the Royal Spanish Academy of Language, to identify it with "a variety of states of pleasant spiritual and physical satisfaction".

Keywords: happiness; gradual pyramid; Maslow

\section{OBJETIVOS Y METODOLOGÍA DEL TRABAJO}

Fig. 1. Cubo de Rubik $3 \times 3$ (fotografía propia).

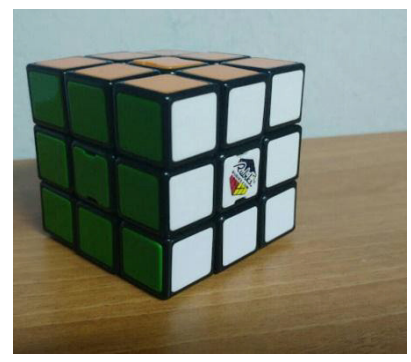

En 1974 Ern Rubik (1944) creó uno de los juguetes más vendidos de la historia. Su Cubo está compuesto por una pieza central esférica oculta a la vista y seis piezas inmóviles equidistantes fijadas en ella, alrededor de las cuales los demás bloques pueden girar sin que se pierda la forma de cubo al observar la estructura externamente (Valenzuela, 2014: 1). Lo cierto es que el Cubo, realmente... ino está formado por cubos! Ninguna de las piezas que lo componen es un cubo, puesto que los ocho vértices (cada uno, de tres colores) y las doce piezas arista (cada una, de dos colores) disponen de un mecanismo no cúbico que permite su giro desde el núcleo.

Para el armador del Cubo de Rubik el reto establecido es conseguir una cara completa de cada color moviendo las piezas que lo conforman. A modo de metáfora, también el ser humano mueve las piezas de su propia vida para llegar a conseguir su felicidad. Podemos preguntarnos entonces ¿puede el ser humano alcanzar la felicidad?

Desde el primer momento advertimos que esta comparativa no es completa, pues si bien el Cubo tiene una solución clara que puede permanecer estática, el objetivo personal del ser humano es más difuso y complejo. También quiero señalar una cuestión metodológica previa. Existen una gran cantidad de análisis que han enfocado su atención en la comprensión de la felicidad de las personas y de las formas para alcanzarla desde puntos de vista muy distintos (estrictamente filosóficos, religiosos, morales, psicológicos, sociológicos...).

En mi primer boceto de ensayo partí de la afirmación de que perseguimos todos un concepto abstracto: la felicidad. Siguiendo las vías estudiadas sobre la forma de alcanzar la felicidad y sus fuentes, entendía sin cuestionarme más que la felicidad debe consistir en algo cercano al advenimiento de una perfección y una armonía monocroma, como la que se puede alcanzar con el Cubo de Rubik. Sin embargo, cuando ya casi había finalizado mi primer ensayo decidí volver atrás y cuestionarme radicalmente la propia posibilidad de consecución de la felicidad, partiendo de una especie de agnosia sobre el concepto de felicidad como estado final. Por ello modifiqué el rumbo del trabajo y lo dirigí hacia la indagación acerca de si realmente es posible alcanzar la felicidad cambiando las piezas y los engranajes de nuestra vida. 


\section{ESTADOS DE LA CUESTIÓN}

Todos nosotros somos lanzados como Cubos de Rubik al mundo con piezas que no podemos modificar y con piezas que sí podremos manejar, con un fin que anhelamos y perseguimos... la felicidad. Usted, cada día, en cada momento elige cómo mueve y coloca sus piezas y su vida en la medida de sus posibilidades, en sus circunstancias como diría Ortega y Gasset (1883-1955) ${ }^{1}$, para llegar a su felicidad. Como decía Stendhal (17831842), cada día salimos "a la caza de la felicidad" (Lalande, 1953: 465). Las ideas homologadas de la felicidad siguen vivas en nuestros días, cada vez más y en más disciplinas, sobre todo en las sociedades occidentales (Bueno, 2011: 80).

Para Aristóteles (384-322 a.C.) "la felicidad y el bien supremo constituyen el verdadero fin de la vida" (Aristóteles, La Gran Moral: 1). Se han dado diversas definiciones de felicidad que podrían condensarse en la obtención de un estado de armonía y de plenitud interior. La felicidad es enriquecedora en cuanto supone un equilibrio 0 un crecimiento interior, lo que coincide con el punto de vista etimológico, pues he observado que las palabras latinas felicitas, felix o feliciter están vinculadas a la raíz indoeuropea dhe(i), que significa mamar 0 amamantar (Treviño Rodríguez, 2018: 1).

Pero ¿y si la felicidad no existe para las personas? ¿Es posible argumentar que la felicidad sea un espejismo o sea inalcanzable? En este sentido Friedrich Wilhelm Nietzsche (1844-1900) sostenía que la felicidad no está ligada a los seres humanos y que no hemos nacido para alcanzarla. Para Nietzsche la felicidad "es un camino inventado por aquellos que mienten y que es impuesto" (Santaolalla, 2016: 1). Según Nietzsche "muchos hombres no son capaces más que de una felicidad insignificante [... ]. Ojalá que todas las personas de buena fortuna encuentren la concepción de la existencia que pueda realizar su más elevada concepción de la felicidad: incluso así su vida puede seguir siendo lamentable y poco envidiable"2. La perspectiva que adopta Nietzsche es peculiar porque ofrece pocas expectativas de llegar a una felicidad significativa. Incluso admitiendo su posición crítica tan pesimista, yo acojo la posibilidad que él mismo admite de llegar a alcanzar alguna forma de felicidad surgida del interior del hombre, por insignificante que sea. Si Nietzsche deja entornada una pequeña ventana a la posibilidad de alcanzar la felicidad, en este trabajo se intentará abrir.

Pero quiero descender un escalón más. Como punto inicial y para no partir de ideas preconcebidas, valoro incluso que estemos persiguiendo una quimera y que la felicidad no sea una palabra categoremática. Como concluye Bueno (1924-2016) cabría negar la Idea de felicidad (Bueno, 2005: 387).

Puedo contraargumentar como base de la discusión que la felicidad no es alcanzable. Pero incluso aunque la usemos simplemente como hipótesis, la idea de felicidad seguirá sirviendo como el norte de la brújula de nuestras vidas, pues como destacó Séneca (4 a. C.-65 d. C.) todos los hombres aspiramos a lograr la felicidad (De vita beata). Partir de la imposibilidad de alcanzar la felicidad nos colocaría ante esa vida lamentable de la que hablaba Nietzsche. La desesperanza total ante la felicidad nos situaría frente a un destino doloroso e insoportable como el reflejado en la obra pictórica "El Grito" de Edvard Munch (1863-1944)33. Así pues, aunque sea como una mera hipótesis, hemos de partir al menos de la posibilidad de perseguir y obtener alguna felicidad en el marco de nuestra conducta individual y de nuestras circunstancias. Aceptaré pues, aunque sea como hipótesis necesaria, que sí existe la felicidad y que es posible alcanzarla. En este caso ¿qué felicidad alcanzaríamos?

\section{DISCUSIÓN Y CONCLUSIONES. CARÁCTER BIFRONTE DE LA FELICIDAD}

Podemos encontrar un aspecto bifronte de la felicidad. Según el Diccionario de la Real Academia Española de la Lengua (RAE, 2017: 1), la felicidad, en su significación primera, es un "estado de grata satisfacción espiritual y física". Quiero llamar la atención sobre el aspecto bifronte de la felicidad. Como se observa, incluso en el lenguaje, una de las palabras más importantes de nuestra vida alude a un estado ambivalente de satisfacción: físico, por un lado, y espiritual por otro. Atenderé a continuación a cada una de estas facetas.

Llevando la felicidad al laboratorio, desde un punto de vista bioquímico la felicidad está correlacionada con el nivel de endorfinas segregadas por nuestra glándula pituitaria y el hipotálamo (Steward, 2000: 116). Desde un 


\section{¿PUEDE El SER HUMANO ALCANZAR LA FELICIDAD?}

punto de vista biológico la felicidad se vincula a la producción de endorfinas, péptidos opioides endógenos que actuarían como neurotransmisores al conseguir la felicidad.

Ciertamente, tanto en el Cubo de Rubik como en la conducta personal se pueden hacer trampas: en el Cubo de Rubik se pueden cambiar las pegatinas de colores de las piezas y conseguir así el resultado anhelado sin seguir los algoritmos requeridos; también el ser humano, artificialmente, mediante opiáceos por ejemplo, puede hacer "trampa" a la felicidad y conseguir temporalmente la segregación masiva de endorfinas para alcanzar una sensación de felicidad. En estos casos no será la felicidad lo que se ha conseguido, sino solo la sensación ficticia y pasajera que produce la felicidad.

Biológicamente sí es posible encontrar un enlace entre felicidad y endorfinas. Sin embargo esta no es una relación bidireccional. No se puede identificar nivel de endorfinas con nivel de felicidad. Hay varios procesos biológicos naturales, como la práctica deportiva, que pueden generar una elevación del nivel de endorfinas sin relación alguna con la felicidad. El componente biológico, por tanto, nos ofrece pistas interesantes que reafirman la hipótesis de que se puede tener más o menos felicidad, aunque no nos ofrece un criterio definitivo sobre su naturaleza 0 su contenido.

Desde el punto de vista espiritual, en cambio, es más difícil acotar el concepto de felicidad y la forma de llegar a ella. Si observamos nuestra propia apercepción de felicidad, si la hemos sentido, comprobamos su fugacidad, su indeterminación y su carácter personalísimo. Nuestra felicidad ha sido, en su caso, como un arco iris: Ilena de belleza, mágica pero evanescente e incapturable. ¿Es que tendemos a la felicidad pero nunca podemos llegar a aprehenderla?

Entiendo que es muy importante diferenciar entre los distintos grados de felicidad espiritual. Me parece que es relevante diferenciar entre ser feliz en mayor o en menor grado y alcanzar la felicidad en el grado máximo. Me referiré ahora a estas dos cuestiones.

Es muy difícil que podamos alcanzar el grado máximo y permanente de felicidad 40 la felicidad completa. Puede que asumamos el peso y la responsabilidad de conseguir la máxima felicidad pero, como Sísifo, cargando una y otra vez la gigantesca piedra hacia la cumbre de la montaña, nunca podamos mantenernos allí, en ese territorio ideal o emocional del que poco conocemos. En este sentido creo que la felicidad en su grado máximo, como felicidad absoluta, es una asíntota, esto es, en geometría, una recta cuya distancia a una curva tiende a cero en el infinito, pero no llega a ese punto 5 .

Con respecto a la posibilidad de ser feliz en mayor o menor medida, es sugerente la propuesta de la Pirámide de Abraham Maslow, que nos indica que podemos ir superando diferentes etapas, proponiéndonos metas distintas, con el fin de mejorar el desarrollo personal y llegar a la autorrealización (García-Allén, 2018: 1). Ciertamente muchas de las críticas que se han vertido sobre esta teoría me parecen razonables: por ejemplo, no ha sido demostrada la jerarquía que Maslow postuló y el concepto de autorrealización es muy abstracto y ambiguo (García-Allén, 2018: 1) ${ }^{6}$. Sin embargo en este momento me parece interesante integrar la idea de la Pirámide de Maslow en el aspecto gradual que realiza para conseguir la felicidad.

Yo propongo salir de ideas preconcebidas sobre el destino de nuestro esfuerzo en pos de la felicidad. Desde mi punto de vista, la felicidad no es lo mismo para todos ni en todos los casos, ni para todos hay una misma forma de felicidad. Pienso que la esencia final de la felicidad será tan diferente para las personas que no habrá un estado de felicidad, sino muchos y muy diferentes estados y grados de satisfacción.

Mi conclusión final es optimista: entiendo que la felicidad relativa engloba un rango muy variable y no es un punto final. Creo que las personas sí podemos alcanzar esta felicidad en nuestra vida. Pienso que en este siglo XXI deberá estudiarse más a fondo cómo será el puerto de llegada, la felicidad, toda vez que ya contamos con guías de distintas rutas éticas que nos pueden permitir alcanzar los distintos grados individuales y temporales de felicidad.

A la vista de esta reflexión entiendo que podría modificarse el significado del término "felicidad" en el Diccionario de la Real Academia Española de la Lengua, para identificarlo con "una variedad de estados de grata satisfacción espiritual y física". 


\section{REFERENCIAS BIBLIOGRÁFICAS}

ARISTÓTELES (s. IV a. C.). "De la felicidad". La Gran Moral. Libro primero. Capítulo IV. Traducción de Patricio Azcárate.

BUENO, G. (2005). El mito de la felicidad. Ediciones B. Barcelona. 2005. Última consulta el 22 de abril de 2020. http://fgbueno.es/med/dig/gb2005f6.pdf

BUENO, G. (2011). "El mito de la felicidad". Tesela, número 80. 26 de octubre de 2011. Última visualización el día 22 de abril de 2020. http://www.fgbueno.es/med/tes/t080.htm

FLEMING, V. (1939). "El Mago de Oz". Estados Unidos. Metro-Goldwyn-Mayer. Escenas finales.

GARCÍA-ALLÉN, J. (2018). "Pirámide de Maslow: la jerarquía de las necesidades humanas". Última consulta el día 22 de abril de 2020. https://psicologiaymente.net/psicologia/piramide-de-maslow

LALANDE, A. (1953). "Sobre felicidad". Vocabulario técnico y crítico de la filosofía. Librería El Ateneo. Buenos Aires, p. 464-465. Última consulta el día 22 de abril de 2020. http://www.filosofia.org/enc/vtc/v1464.htm

MUNCH, E. (1893). "El Grito".

NIETZSCHE, F. (2000). Aurora. Reflexiones sobre los prejuicios morales. Traducción Germán Cano. Madrid: Biblioteca Nueva, primera edición, p. 108 y 345.

ORTEGA Y GASSET, J. (1914). Meditaciones del Quijote. Revista de Occidente, Madrid, p. 16.

PÉREZ PORTO, J. y GARDEY, A. (2015), "Definición de asíntota". Última consulta el día 22 de abril de 2020. https://definicion.de/asintota/

REAL ACADEMIA ESPAÑOLA (2017). "Felicidad". Diccionario de la Lengua Española. Vigesimotercera edición actualizada en 2017. Última consulta el día 22 de abril de 2020. http://dle.rae.es/?id=Hj4JtKk

SANTAOLALLA, G. (2016). "La felicidad para Nietzsche". Última consulta el día 22 de abril de 2020. http://www.dialogoexistencial.com/la-felicidad-para-nietzsche/

STEWARD, 0. (2000). Functional Neuroscience. Springer Science \& Business Media. University of California, p. 116.

TREVIÑO RODRíGUEZ, J. (2018). "Etimología de felicidad", p. 1. Última consulta el día 6 de enero de 2018. http://etimologias.dechile.net/?felicidad

VALENZUELA, I. (2014). "Conoce la sorprendente historia detrás del Cubo de Rubik". Última consulta el día 22 de abril de 2020. https://www.vix.com/es/btg/curiosidades/7114/conoce-la-sorprendente-historia-detrasdel-cubo-de-rubik

\section{NOTAS}

1 "Yo soy yo y mi circunstancia, y si no la salvo a ella no me salvo yo", dijo Ortega y Gasset en sus Meditaciones del Quijote (Ortega y Gasset, 1914: 16).

2 Nietzsche, F. (1881), Aurora. Reflexiones sobre los prejuicios morales. Biblioteca Nueva, 2000 (Santaolalla, 2016:1).

3 Much, 1893: "El Grito".

4 En este trabajo siempre se habla exclusivamente de la consecución de la felicidad en la Tierra en vida. En este sentido se acogen las palabras de una de las escenas finales de la película "El Mago de Oz" (Victor Fleming, 1939): "se está mejor en casa que en ningún sitio".

5 El término griego asíntota hace referencia a algo que no tiene coincidencia, y en geometría nombra una recta que, a medida que se prolonga de manera indefinida, tiende a acercarse a una curva o función, aunque sin alcanzar a hallarla (Pérez y Gardey, 2015:1).

6 En un estudio publicado en 2011, investigadores de la Universidad de Illinois descubrieron que la satisfacción de las necesidades de la pirámide correlacionaba con la felicidad de la persona pero se cuestionaba la secuencialidad que Maslow propuso en su teoría (García-Allén, 2018:1 
